\title{
ON THE IRREDUGIBILITY OF CONVEX BODIES
}

\author{
A. C. WOODS
}

1. Introduction. We select a Cartesian co-ordinate system in $n$ dimensional Euclidean space $R_{n}$ with origin $O$ and employ the usual pointvector notation.

By a lattice $\Lambda$ in $R_{n}$ we mean the set of all rational integral combinations of $n$ linearly independent points $X_{1}, X_{2}, \ldots, X_{n}$ of $R_{n}$. The points $X_{1}, X_{2}, \ldots$, $X_{n}$ are said to form a basis of $\Lambda$. Let $\left\{X_{1}, X_{2}, \ldots, X_{n}\right\}$ denote the determinant formed when the co-ordinates of $X_{i}$ are taken in order as the $i$ th row of the determinant for $i=1,2, \ldots, n$. The absolute value of this determinant is called the determinant $d(\Lambda)$ of $\Lambda$. It is well known that $d(\Lambda)$ is independent of the particular basis one takes for $\Lambda$.

A star body in $R_{n}$ is a closed set of points $K$ such that if $X \in K$ then every point of the form $t X$ where $-1<t<1$ is an inner point of $K$. A star body $K$ is called a convex body if it is bounded and satisfies the convex property: if $X \in K, Y \in K$ then $t X+(1-t) Y \in K$ provided $0 \leqq t \leqq 1$. It is further called strictly convex if $X \in K, Y \in K$ implies that $t X+(1-t) Y$ is an inner point of $K$ when $0<t<1$ and $X \neq Y$.

Let $\Lambda$ be a lattice and $K$ a star body in $R_{n}$. We say that $\Lambda$ is $K$-admissible if no point of $\Lambda$ other than 0 is an inner point of $K$. If $K$ is such that no $K$ admissible lattice exists then $K$ is said to be of the infinite type, otherwise $K$ is said to be of the finite type. If $K$ is of the finite type the number inf $d(\Lambda)$ extended over all $K$-admissible lattices $\Lambda$ is called the critical determinant $\Delta(K)$ of $K$ and any $K$-admissible lattice $\Lambda$ of determinant $d(\Lambda)=\Delta(K)$ is called a critical lattice of $K$. It is well known that if $K$ is of the finite type then at least one critical lattice of $K$ exists.

Let $K$ be a star body of the finite type in $R_{n}$. If $K$ is such that any star body properly contained in $K$ has a smaller critical determinant than $K$ has we say that $K$ is $S$-irreducible; otherwise $K$ is said to be $S$-reducible.

Let $K$ be a convex body in $R_{n}$. If $K$ is such that any convex body properly contained in $K$ has a smaller critical determinant than $K$ has then we say that $K$ is $C$-irreducible; otherwise we say that $K$ is $C$-reducible.

The property of $S$-irreducibility was first studied by Mahler (1) who gave necessary but insufficient conditions for a star body to be $S$-irreducible. Later (2) he considered the property of $C$-irreducibility and showed that if $n=2$ then any $C$-irreducible convex body is also $S$-irreducible. Rogers (5) then gave a set of necessary and sufficient conditions for $S$-irreducibility which will be stated later.

Received July 3, 1958. 
The purpose here is to give an example of a convex body in $R_{3}$ that is $C$-irreducible but not $S$-irreducible. The proof that the example has these properties relies to a large extent on the work of Whitworth (6). To clarify the picture regarding $C$-irreducibility we formulate a set of necessary and sufficient conditions for $C$-rreducibility analogous to the set given by Rogers for $S$-irreducibility, the proof following similar lines.

2. The set $L(K)$. The results stated in this section are classical.

Let $K$ be a convex body in $R_{n}$. We define $L(K)$ to be the set of all points $X$ of the boundary of $K$ such that if $X$ is contained in any line segment of the boundary of $K$ then $X$ is an endpoint of the line segment. Such points are sometimes called extremal points of $K$ so that $L(K)$ constitutes the set of all extremal points of $K$. As $K$ is symmetric in 0 it is evident that $L(K)$ is also symmetric in 0 . Further:

Lemma 1. The convex hull of $L(K)$ is $K$.

Lemma 2. Given $X \in L(K)$ and $\epsilon>0$ there exists a convex body $K(\epsilon) \subset K$ such that $X \notin K(\epsilon)$ and such that any point of $K-K(\epsilon)$ lies within a distance $\epsilon$ of one of the two points $\pm X$.

3. $C$-irreducibility. Let $K$ be a star body in $R_{n}$. Further let $\Lambda$ be a critical lattice of $K$. Let $X$ be a point of $\Lambda$ on the boundary of $K$. We say that $\Lambda$ is free at the point $X$ if, given $\epsilon>0$, there exists a lattice $\Lambda(\epsilon)$ of determinant $d(\Lambda(\epsilon))<d(\Lambda)=\Delta(K)$ such that the interior of $K$ contains no point of $\Lambda(\epsilon)$ apart from 0 and any that are within a distance $\epsilon$ from one of the two points $\pm X$. Rogers' criterion for $S$-irreducibility is then as follows:

LEMмA $3 . K$ is S-irreducible if, and only if, to each point of the boundary of $K$ there corresponds a critical lattice of $K$ that is free at this point.

We now give an analogous criterion for $C$-irreducibility.

Theorem 1. If $K$ is a convex body then $K$ is $C$-irreducible if, and only if, to each point of $L(K)$ there corresponds a critical lattice of $K$ that is free at this point.

Proof. (i) Only if: Assume that $K$ is $C$-irreducible and let $X$ be an arbitrary point of $L(K)$. By Lemma 2 given $\epsilon>0$ there exists a convex body $K(\epsilon) \subset K$ such that $X \in K-K(\epsilon)$ and such that any point of $K-K(\epsilon)$ is within a distance $\epsilon$ from one of the two points $\pm X$. Since $K(\epsilon)$ is properly contained in $K$ it follows that $\Delta(K(\epsilon))<\Delta(K)$. Hence there exists a critical lattice $\Lambda(\epsilon)$ of $K(\epsilon)$ of determinant $d(\Lambda(\epsilon))<d(\Lambda)$. It is evident that $K$ contains no point of $\Lambda(\epsilon)$ in its interior other than 0 and any that may lie within a distance $\epsilon$ from one of the two points $\pm X$. Moreover $\Lambda(\epsilon)$ is certainly not $K$-admissible and therefore taking into account the fact that $K$ is symmetric in 0 we conclude that there must be a point of $\Lambda(\epsilon)$ in the interior of $K$ and 
within a distance $\epsilon$ from the point $X$. The sequence $\Lambda\left(n^{-1}\right)$ of lattices is compact in the sense of Mahler (3) and so contains a convergent subsequence with the limit $\Lambda^{\prime}$ say. But $\lim _{n \rightarrow \infty} K\left(n^{-1}\right)=K$ and $\Lambda\left(n^{-1}\right)$ is a critical lattice of $K\left(n^{-1}\right)$ for each $n$, hence $\Lambda^{\prime}$ is a critical lattice of $K$. Further each $\Lambda\left(n^{-1}\right)$ contains a point within a distance $n^{-1}$ from the point $X$. Thus $\Lambda^{\prime}$ contains $X$ which implies that $\Lambda^{\prime}$ is free at $X$. As $X$ was chosen an arbitrary point of $L(K)$ this proves (i).

(ii) If: Assume that to each point of $L(K)$ there corresponds a critical lattice of $K$ that is free at this point. Take an arbitrary convex body $K^{\prime} \subset K$ such that $K^{\prime} \neq K$. There exists a point $X \in L(K)-K^{\prime}$ for otherwise $L(K) \subset K^{\prime}$ and so by Lemma $1 K^{\prime}=K$ contrary to hypothesis. Let $X \in L(K)$ $-K^{\prime}$ be fixed. As $K^{\prime}$ is closed there exists $\epsilon>0$ such that no point within a distance $\epsilon$ from either of the two points $\pm X$ is in $K^{\prime}$. By hypothesis there exists a critical lattice $\Lambda$ of $K$ such that $\Lambda$ is free at the point $X$. In particular this implies that there exists a lattice of determinant $d(\Lambda(\epsilon))<d(\Lambda)=\Delta(K)$ such that no point of $\Lambda(\epsilon)$ apart from 0 and any that may lie within a distance $\epsilon$ from one of the two points $\pm X$ is an inner point of $K$. Hence $\Lambda(\epsilon)$ is $K^{\prime}$ admissible from which it follows that $\Delta\left(K^{\prime}\right) \leqq d(\Lambda(\epsilon))<\Delta(K)$. Whence $K$ is $C$-irreducible. This completes the proof of the theorem.

4. An Example. In looking for a convex body that is $C$-irreducible and $S$-reducible we may by Mahler's result confine our attention to dimensions $n \geqq 3$. Further if $K$ is a strictly convex body it is obvious that $L(K)$ is the whole boundary of $K$. Hence using the previous results $K$ is $C$-irreducible if, and only if, it is $S$-irreducible. Again, Dr. Kathleen Ollerenshaw has obtained the following two results (4):

(a) The $n$-dimensional parallelopiped is $S$-irreducible for every $n$.

(b) If $K$ is a two-dimensional $S$-irreducible convex body then the threedimensional cylinder on the base $K$ is also $S$-irreducible.

A more suitable candidate for our purpose has proved to be a sawn-off three-dimensional cube. Whitworth (6) has shown that the convex body $K$ in $R_{3}$ defined by the inequalities

$$
\left|x_{1}\right| \leqq 1, \quad\left|x_{2}\right| \leqq 1, \quad\left|x_{3}\right| \leqq 1, \quad\left|x_{1}+x_{2}+x_{3}\right| \leqq \frac{1}{2}
$$

has the critical determinant $\Delta(K)=3 / 8$. He has further determined all the critical lattices of $K$. It is necessary to give a table of these here but before doing so we remark that $K$ has the six automorphisms obtained by permuting the co-ordinates together with the reflections in 0 . Thus given any critical lattice of $K$ we obtain six when we apply these transformations. In the following table the only critical lattices of $K$ not included are those obtainable from the ones stated by applying the above automorphisms of $K$. There are three classes:

Class I: $\Lambda(\rho, \sigma, \beta)$ of basis $X_{1}=\left(\rho-\frac{1}{2}, \sigma-1, \beta\right), X_{2}=\left(\rho, \sigma-\frac{1}{2}, \beta-1\right)$, $X_{3}=\left(\rho-1, \sigma, \beta-\frac{1}{2}\right)$ where $\rho+\sigma+\beta=2$. Another basis for $\Lambda(\rho, \sigma, \beta)$ 
would be $X_{2}, X_{2}-X_{1}=\left(\frac{1}{2}, \frac{1}{2},-1\right), X_{3}-X_{2}=\left(-1, \frac{1}{2}, \frac{1}{2},\right)$. The points $X_{2}-X_{1}, X_{3}-X_{1}$ lie in the plane $x_{1}+x_{2}+x_{3}=0$ while $X_{2}$ lies in the plane $x_{1}+x_{2}+x_{3}=\frac{1}{2}$. Hence all points of $\Lambda(\rho, \sigma, \beta)$ that lie on the boundary of $K$ are confined to the three planes $x_{1}+x_{2}+x_{3}=0$ or $\pm 1 / 2$. It follows that the same is true of the automorphic images of $\Lambda(\rho, \sigma, \beta)$.

Class II: $\Lambda(\lambda, \mu, \beta)$ of basis $X_{1}=\left(1,-\frac{1}{2},-\frac{1}{2}\right), X_{2}=\left(-\frac{1}{2}, 1,-\frac{1}{2}\right)$, $X_{3}=(-\lambda,-\mu, \beta)$ where $\lambda+\mu-\beta=\frac{1}{2}, 0<-\beta \leqq \frac{1}{2}, 0 \leqq \mu \leqq \frac{1}{2}$, $0 \leqq \lambda \leqq \frac{1}{2}$. The points $X_{1}, X_{2}$ lie in the plane $x_{1}+x_{2}+x_{3}=0$ while the point $X_{3}$ lies in the plane $x_{1}+x_{2}+x_{3}=-\frac{1}{2}$. Hence all points of $\Lambda(\lambda, \mu, \beta)$ that lie on the boundary of $K$ are confined to the three planes $x_{1}+x_{2}+x_{3}=0$ or $\pm \frac{1}{2}$ and the same is true of the automorphic images of $\Lambda(\lambda, \mu, \beta)$.

Class III: (i) $\Lambda\left(\nu_{1}, \nu_{2}, \chi_{1}, \chi_{2}, \beta\right)$ of basis $X_{1}=\left(-\nu_{1}, \beta,-\chi_{1}\right), X_{2}=$ $\left(-\nu_{2}, 1-\beta,-\chi_{2}\right), X_{3}=\left(1,-\frac{1}{2},-\frac{1}{2}\right)$ where $\nu_{1}+\nu_{2}=\frac{1}{2}, \chi_{1}+\chi_{2}=\frac{1}{2}$, $\beta-\nu_{1}-\chi_{1}= \pm \frac{1}{2}$. The points $X_{1}, X_{2}$ lie in one of the planes $x_{1}+x_{2}+x_{3}$ $= \pm \frac{1}{2}$ while the point $X_{3}$ lies in the plane $x_{1}+x_{2}+x_{3}=0$ and hence all points of $\Lambda\left(\nu_{1}, \nu_{2}, \chi_{1}, \chi_{2}, \beta\right)$ that are on the boundary of $K$ are confined to the planes $x_{1}+x_{2}+x_{3}=0$ or $\pm \frac{1}{2}$.

(ii) $\Lambda(\lambda)$ of basis $X_{1}=\left(1,-\frac{1}{2},-\frac{1}{2}\right), X_{2}=\left(-\lambda,-\frac{1}{2}, 1\right), X_{3}=\left(\frac{1}{2}, 0,0\right)$. Evidently the points of $\Lambda(\lambda)$ that are on the boundary of $K$ are confined to the lines given by $\left(t,-\frac{1}{2} u_{1}-\frac{1}{2} u_{2},-\frac{1}{2} u_{1}+u_{2}\right)$ where $u_{1}, u_{2}$ have one of the following pairs of values: $(0,0),(1,0),(-1,0),(0,1),(0,-1),(1,1)$, $(-1,-1),(2,0),(-2,0)$. Hence the points of all the automorphic images of $\Lambda(\lambda)$ on the boundary of $K$ are confined to the lines given above together with those obtained from them by permuting the co-ordinates.

(iii) $\Lambda$ of basis $X_{1}=\left(-\frac{1}{2}, 1,-\frac{1}{2}\right), X_{2}=\left(\frac{1}{2},-\frac{1}{2},-\frac{1}{2}\right), X_{3}=\left(\frac{1}{2}, 0,0\right)$. The point $X_{1}$ lies in the plane $x_{1}+x_{2}+x_{3}=0, X_{2}$ in $x_{1}+x_{2}+x_{3}=-\frac{1}{2}$, $X_{3}$ in $x_{1}+x_{2}+x_{3}=\frac{1}{2}$; hence all points of $\Lambda$ that are on the boundary of $K$ are confined to the planes $x_{1}+x_{2}+x_{3}=0$ or $\pm \frac{1}{2}$. It follows that the same is true of the automorphic images of $\Lambda$.

This completes the table of the critical lattices of $K$. We are now in a position to prove:

\section{Theorem 2. $K$ is C-irreducible and S-reducible.}

Proof. We show first that $K$ is $S$-reducible. From the table given above we see that the only critical lattices of $K$ with points on the boundary of $K$ that do not lie in one of the three planes $x_{1}+x_{2}+x_{3}=0$ or $\pm \frac{1}{2}$ are those in Class III (ii). The point $\left(1,-\frac{1}{3},-\frac{1}{3}\right)$ is on the boundary of $K$ and in the plane $x_{1}+x_{2}+x_{3}=\frac{1}{3}$. Therefore if it is a point of some critical lattice of $K$ it must be in Class III (ii). However, it is obvious that no lattice of this class can contain $\left(1,-\frac{1}{3},-\frac{1}{3}\right)$ nor can any lattice which is derived from one of those stated by permuting the co-ordinates. Therefore $\left(1,-\frac{1}{3},-\frac{1}{3}\right)$ belongs to no critical lattice of $K$. By Lemma $3, K$ is $S$-reducible. 
We now show that $K$ is $C$-irreducible. The set $L(K)$ consists of the twelve points obtained by permuting the co-ordinates of the point $\left(1, \frac{1}{2},-1\right)$ and taking the six points thus obtained together with their reflections in 0 . Hence, by virtue of Theorem $1, K$ is $C$-irreducible if we can show that there exists a critical lattice of $K$ which is free at the point $\left(1, \frac{1}{2},-1\right)$. Take the lattice $\Lambda\left(\frac{1}{2}, 0,3 / 2\right)$ in Class I of the table above. A basis of this lattice is $X_{1}=(1$, $\left.-1, \frac{1}{2}\right), X_{2}=\left(3 / 2,-\frac{1}{2},-\frac{1}{2}\right), X_{3}=\left(\frac{1}{2}, 0,0\right)$. Another basis would be $Y_{1}=X_{2}-X_{1}=\left(\frac{1}{2}, \frac{1}{2},-1\right), Y_{2}=X_{1}-X_{3}=\left(\frac{1}{2},-1, \frac{1}{2}\right), Y_{3}=X_{3}$. The points of $\Lambda\left(\frac{1}{2}, 0,3 / 2\right)$ on the boundary of $K$ are $Y_{1}, Y_{2}, Y_{3}, Y_{1}+Y_{2}=(1$, $\left.-\frac{1}{2},-\frac{1}{2}\right), Y_{1}+Y_{3}=\left(1, \frac{1}{2},-1\right), Y_{1}-Y_{3}=\left(0, \frac{1}{2},-1\right), Y_{2}-Y_{3}=(0$, $\left.-1, \frac{1}{2}\right), \quad Y_{2}+Y_{3}=\left(1,-1, \frac{1}{2}\right), \quad Y_{1}+Y_{2}-Y_{3}=\left(\frac{1}{2},-\frac{1}{2},-\frac{1}{2}\right)$ together with their reflections in 0 . In particular we see that $Y_{1}+Y_{3}=\left(1, \frac{1}{2},-1\right)$ is a point of the lattice. For a given $\delta>0$ denote by $\Lambda(\delta)$ the lattice of basis $Y_{1}^{\prime}=\left(\frac{1}{2}-\delta, \frac{1}{2},-1\right), Y_{2}^{\prime}=\left(\frac{1}{2}+\delta,-1, \frac{1}{2}\right), Y_{3}^{\prime}=\left(\frac{1}{2}-\delta, 0, \delta\right)$. Evidently as $\delta \rightarrow 0$ so $Y_{1}^{\prime} \rightarrow Y_{1}, Y_{2}^{\prime} \rightarrow Y_{2}, Y_{3}{ }^{\prime} \rightarrow Y_{3}$ and therefore also $\Lambda(\delta) \rightarrow \Lambda\left(\frac{1}{2}, 0\right.$, $3 / 2)$. Moreover,

$$
d(\Lambda(\delta))=\left\|\begin{array}{rrr}
\frac{1}{2}-\delta & \frac{1}{2} & -1 \\
\frac{1}{2}+\delta & -1 & \frac{1}{2} \\
\frac{1}{2}-\delta & 0 & \delta
\end{array}\right\|=\frac{3}{8}-\frac{1}{2} \delta^{2}<\frac{3}{8}
$$

provided only that $\delta$ is sufficiently small. Since in the limit $\delta \rightarrow 0$ the basis given for $\Lambda(\delta)$ becomes the basis given for $\Lambda\left(\frac{1}{2}, 0,3 / 2\right)$ it follows that for all sufficiently small $\delta$ the only points of $\Lambda(\delta)$ that can lie in the interior of $K$ are

$$
\begin{aligned}
& Y_{1}^{\prime}=\left(\frac{1}{2}-\delta, \frac{1}{2},-1\right), Y_{2}^{\prime}\left(\frac{1}{2}+\delta,-1, \frac{1}{2}\right), Y_{3}^{\prime}=\left(\frac{1}{2}-\delta, 0, \delta\right), \\
& Y_{1}^{\prime}+Y_{2}^{\prime}=\left(1,-\frac{1}{2},-\frac{1}{2}\right), Y_{1}^{\prime}+Y_{3}^{\prime}=\left(1-2 \delta, \frac{1}{2}, \delta-1\right), Y_{2}^{\prime}+Y_{3}^{\prime} \\
& =\left(1,-1, \frac{1}{2}+\delta\right) \text {, } \\
& Y_{1}^{\prime}-Y_{3}^{\prime}=\left(0, \frac{1}{2},-1-\delta\right), Y_{2}^{\prime}-Y_{3}^{\prime}=\left(2 \delta,-1, \frac{1}{2}-\delta\right), Y_{1}^{\prime}+Y_{2}^{\prime}-Y_{3}^{\prime} \\
& =\left(\frac{1}{2}+\delta,-\frac{1}{2},-\frac{1}{2}-\delta\right)
\end{aligned}
$$

together with their reflections in 0 . But it is clear that the only ones in the interior of $K$ are $\pm\left(Y_{1}{ }^{\prime}+Y_{3}{ }^{\prime}\right)$. Moreover

$$
\lim _{\delta \rightarrow R}\left(Y_{1}^{\prime}+Y_{3}^{\prime}\right)=\left(1, \frac{1}{2},-1\right),
$$

hence $\Lambda\left(3 / 2,0, \frac{1}{2}\right)$ is free at the point $\left(1, \frac{1}{2},-1\right)$. Therefore $K$ is $C$-irreducible. This completes the proof of Theorem 2 .

Part of this work is extracted from a thesis for the degree of Doctor of Philosophy at the University of Manchester, written under the supervision of Professor K. Mahler to whom I am very grateful for advice and encouragement. 


\section{REFERENCES}

1. K. Mahler, Lattice points in n-dimensional star bodies II, Reducibility theorems, Proc. Nederl. Akad. Wetensch., 49 (1946), 331-43.

2. - On irreducible convex domains, Proc. Nederl. Akad. Wetensch., 50 (1947), 98-107.

3. Lattice points in n-dimensional star bodies, I, Existence theorems, Proc. Roy. Soc. London, Ser. A, 187 (1946), 151-87.

4. K. Ollerenshaw, Irreducible convex bodies, Quart. J. Math., Oxford (2), 4 (1953), 293-302.

5. C. A. Rogers, $A$ note on irreducible star bodies, Proc. Nederl. Akad. Wetensch., 50 (1947), 868-72.

6. J. V. Whitworth, On the densest packing of sections of a cube, Ann. Mat. Pura Appl., Ser. 4, 27 (1948), 29-37.

Tulane University of Louisiana 